\title{
Materiais didáticos utilizados nas formações de professores em Pensamento Computacional
}

\author{
Soraya Roberta dos Santos Medeiros ${ }^{1}$, Cibelle Amorim Martins ${ }^{1,2}$, Inácio Gomes \\ Medeiros $^{3}$
}

${ }^{1}$ Programa de Pós-Graduação em Inovação em Tecnologias Educacionais - Instituto Metrópole Digital - Universidade Federal do Rio Grande do Norte (UFRN)

Caixa Postal 1524 - 59078-970 - Natal - RN - Brasil

${ }^{2}$ Centro de Educação

Universidade Federal do Rio Grande do Norte (UFRN) - Natal, RN - Brazil

${ }^{3}$ Instituto do Cérebro

Universidade Federal do Rio Grande do Norte (UFRN) - Natal, RN - Brazil

soraya.roberta.js@gmail.com, cibelle.amorimeufrn.br,

inacio.medeiros@neuro.ufrn.br

\begin{abstract}
This article presents the results from a systematic literacy mapping that investigates the availability of teaching materials used in teachers training with Computational Thinking (CT). The main 2015-2019 English and Portuguese-written CT papers bases were selected. Analysis revealed that digital tools like Scratch and Lightboot are the most used and that CT teaching is restricted to project learning and development. Thus, this work evidenced that this is a new area with many gaps to be studied, specially for teachers that act, for example, on Young and Adults of Education.
\end{abstract}

Resumo Este estudo apresenta os resultados de um mapeamento sistemático da literatura que investigou a presença de materiais didáticos utilizados nas formações de professores em Pensamento Computacional (PC). Foram selecionadas as principais bases de pesquisa que possuem publicações relacionadas ao PC escritas em português e inglês entre os anos de 2015-2019. A análise revelou que as ferramentas digitais Scratch e Lightboot são as mais utilizadas e que o ensino de PC está restrito a aprender a desenvolver projetos. Assim, a pesquisa demonstrou que esta é uma área nova, na literatura, com muitas lacunas a serem estudadas, principalmente para professores que atuam em modalidades de ensino como a Educação de Jovens e Adultos.

\section{Introdução}

Desenvolver habilidades para atuar de forma mais criativa e crítica no mercado de trabalho, na realização de tarefas cotidianas ou no desempenho de atividades escolares, tem se tornado uma necessidade cada vez mais discutida em fóruns econômicos (WEF 2020) e congressos educacionais (CBIE 2020). A fim de atender a essas necessidades, o Pensamento Computacional vem despontando como um importante conceito nesse cenário. O termo, que surgiu na área da Computação, é ressaltado e reafirmado como válido a ser desenvolvido não apenas por Cientistas da Computação, mas por diversas áreas e profissões (WING, 2006). Isso ocorre porque, a partir da sua prática, o ser humano consegue analisar suas tarefas e fragmentá-la em algumas etapas, a saber: abstração, 
decomposição, reconhecimento de padrões e algoritmos (Brackman 2017).

Dadas essas etapas do PC, ele permite que seja construído uma pedagogia como prática para a liberdade, tal como propôs Paulo Freire, pois, ao aplicá-la, o que se está desenvolvendo é a práxis, ação e reflexão, concomitantemente. Portanto, faz-se necessário que o PC faça parte também da formação de professores. Se isso acontecer, tornar-se-á mais comum a existência de sujeitos tomando decisões com base em ações coerentes e críticas, pois o papel do professor é orientar o conhecimento de seus alunos de forma sistematizada (Freire 2005).

É notório o crescimento do número de materiais que são produzidos para alunos, principalmente da Educação Básica, trabalhar PC em sala de aula (Medeiros, Martins, Madeira, 2020; Schulz, Schmachtenberg 2017; Bell et al. 2011), contudo, não se pode afirmar que exista um mesmo movimento para professores. Nesse sentido, este estudo busca investigar a utilização de materiais didáticos nas formações de professores em Pensamento Computacional. Logo, torna-se salutar a existência de trabalhos como este mapeamento, uma vez que podem contribuir para a elucidação de características e lacunas existentes na literatura, permitindo, assim, uma personalização de acordo com as necessidades apontadas.

Este artigo está dividido nos seguintes tópicos: esta parte introdutória, que apresenta o objetivo da pesquisa e sua importância para a formação de professores com Pensamento Computacional; metodologia, que descreve o processo metodológico da pesquisa realizada, contemplando o processo de busca, a construção da string utilizada nas bases, as questões de pesquisa, bem como a sistematização dos seus critérios de inclusão e exclusão para análise dos trabalhos selecionados; resultados e discussões, que visam responder às questões de pesquisa, seguidos das considerações finais e referências.

\section{Metodologia}

Um mapeamento sistemático permite expor uma visão geral e apontar oportunidades e desafios que podem ser desenvolvidos em determinada área de pesquisa por meio da literatura. Também possibilita elencar onde esses estudos estão sendo publicados (Kitchenham, Charters 2010; Kitchenham et al. 2010). Para a realização deste estudo foram utilizadas as orientações metodológicas apontadas em Petersen, Vakkalanka e Kuzniarz (2015).

Assim, para desenvolvê-lo, foram construídas questões de pesquisa, elaboração de um protocolo a ser seguido, por meio da construção de strings de buscas, sendo adaptadas para os idiomas pesquisados e suas respectivas bases de busca. Posteriormente, foram explicitados os critérios de inclusão e exclusão que orientam e delimitam o que se deve considerar para realizar um levantamento dos trabalhos mapeados. Além disso, é apresentado um critério de qualidade e um debruçamento sobre os trabalhos retornados nas buscas com vistas a responder às questões de pesquisa (Petersen, Vakkalanka, Kuzniarz 2015). 


\subsection{Processo de busca}

\section{Quadro 1. Protocolo PICO}

\begin{tabular}{|c|l|}
\hline ID & \multicolumn{1}{|c|}{ Research Question } \\
\hline RQ1 & $\begin{array}{l}\text { Qual o contexto, período, coleta de dados e avaliação empregada nas práticas de formação de } \\
\text { professores com Pensamento Computacional? }\end{array}$ \\
\hline RQ2 & $\begin{array}{l}\text { Quais são os materiais didáticos que estão sendo adotados para formar professores para atuar } \\
\text { com Pensamento Computacional? }\end{array}$ \\
\hline RQ3 & $\begin{array}{l}\text { Quais os níveis de ensino que atuam os professores que estão utilizando esses materiais didáticos } \\
\text { nessas pesquisas? }\end{array}$ \\
\hline RQ4 & $\begin{array}{l}\text { Existe algum material didático para formar professores para atuar com Pensamento } \\
\text { Computacional em alguma modalidade de ensino nessas pesquisas? }\end{array}$ \\
\hline
\end{tabular}

Population: Artigos científicos que falem sobre formação de professores e Pensamento Computacional. Intervention: materiais adotados para formar professores para atuar com Pensamento Computacional. Comparasion: Classificar as publicações de acordo com o nível e modalidade de ensino. Outcomes: Definição dos materiais adotados para formar professores de acordo com o nível e modalidade de ensino que ele atua. Keywords: Formação de professores e Pensamento Computacional.

\section{Quadro 2. Strings de busca utilizadas}

\begin{tabular}{|c|l|}
\hline Idioma & \multicolumn{1}{|c|}{ String de busca básica } \\
\hline Português & $\begin{array}{l}\text { ("formação docente” OR “formação de professor" OR “formação de professores") AND } \\
\text { ("pensamento computacional” OR "computational thinking”) }\end{array}$ \\
\hline Inglês & $\begin{array}{l}\text { ("tranning teacher" OR "formação de professor" OR "formacao de professores") AND (CT } \\
\text { OR "Computational Thinking") }\end{array}$ \\
\hline
\end{tabular}

\subsection{Bases de dados}

A estratégia do processo de pesquisa foi uma busca por artigos, dissertações ou teses publicados nas seguintes bases de dados: Revista Brasileira de Informática na Educação (RBIE), Anais dos Workshops do Congresso Brasileiro de Informática na Educação (WIE/CBIE) e do Simpósio Brasileiro de Informática na Educação (SBIE/CBIE), Revista Novas Tecnologias da Educação (Renote), Biblioteca Digital de Teses e Dissertações (BDTD) da Capes, ACM.

Quadro 3. Strings de busca refinadas por base de dados

\begin{tabular}{|c|c|}
\hline Base & String de busca refinada \\
\hline RENOTE & $\begin{array}{l}\text { ("formação docente" OR "formação de professor" OR "formacao de professores") AND } \\
\text { ("pensamento computacional" OR "Computational Thinking") }\end{array}$ \\
\hline SBIE & $\begin{array}{l}\text { ("formação docente" OR "formação de professor" OR "formacao de professores") AND } \\
\text { ("pensamento computacional" OR "Computational Thinking") }\end{array}$ \\
\hline
\end{tabular}




\begin{tabular}{|c|c|}
\hline WCBIE & $\begin{array}{l}\text { ("formação docente" OR "formação de professor" OR "formacao de professores") AND } \\
\text { ("pensamento computacional" OR "Computational Thinking") }\end{array}$ \\
\hline WIE & $\begin{array}{l}\text { ("formação docente" OR "formação de professor" OR "formacao de professores") AND } \\
\text { ("pensamento computacional" OR "Computational Thinking") }\end{array}$ \\
\hline RBIE & $\begin{array}{l}\text { ("formação docente" OR "formação de professor" OR "formacao de professores") AND } \\
\text { ("pensamento computacional" OR "Computational Thinking") }\end{array}$ \\
\hline BDTD & $\begin{array}{l}(\text { formação AND (docente* OR professor*)) AND ("pensamento computacional" OR } \\
\text { "Computational Thinking"))" }\end{array}$ \\
\hline IEEE & (“Teacher training") AND ("Computational Thinking") \\
\hline $\mathrm{ACM}$ & $\begin{array}{l}\text { "teachers education" OR "teacher education" OR "professor training"] OR [All: "teacher } \\
\text { training"]] AND [All: "computational thinking"] }\end{array}$ \\
\hline
\end{tabular}

\section{Quadro 4. Critérios de seleção adotados}

\begin{tabular}{|c|c|l|}
\hline \multirow{2}{*}{ Inclusão } & ID & \multicolumn{1}{|c|}{ Descrição } \\
\cline { 2 - 3 } & I1 & Trabalhos que foram publicados entre 2015 e 2019 \\
\cline { 2 - 3 } & I2 & $\begin{array}{l}\text { Trabalhos publicados em periódicos, revistas e congressos } \\
\text { científicos ou dissertações e teses }\end{array}$ \\
\cline { 2 - 3 } & I3 & $\begin{array}{l}\text { Trabalhos que foram escritos em Língua Portuguesa ou } \\
\text { inglesa }\end{array}$ \\
\hline \multirow{2}{*}{ Exclusão } & I4 & $\begin{array}{l}\text { Trabalhos que discutem sobre formação de professores para } \\
\text { atuar com Pensamento Computacional }\end{array}$ \\
\cline { 2 - 3 } & E1 & Trabalhos duplicados \\
\cline { 2 - 3 } & E2 & $\begin{array}{l}\text { Revisões de literatura ou outros trabalhos que não discutam } \\
\text { sobre intervenções práticas com os professores }\end{array}$ \\
\cline { 2 - 3 } & E3 & Pesquisas que não disponibilizam o texto completo \\
\hline
\end{tabular}

\subsection{Critérios de verificação da qualidade dos estudos}

Trabalhos que não especificam os níveis de ensino que atuam os professores que participaram das formações.

\section{Resultados e discussões}

A fim de compreender o estado da arte dos estudos sobre formação de professores para atuar com PC, foi realizado um Mapeamento Sistemático de Literatura. Quando as respostas às questões de pesquisa não foram explícitas pelos autores, assumiu-se que não foram encontradas informações suficientes, portanto, o campo a ser preenchido conta com a informação "NÃO DESCREVE". 
Quadro 5. Trabalhos analisados neste artigo.

\begin{tabular}{|c|c|c|}
\hline Citação & Referência bibliográfica & ID \\
\hline $\begin{array}{l}\text { Barros et al. } \\
(2018)\end{array}$ & $\begin{array}{l}\text { Barros, Taise T. T.; Reategui, Eliseo Berni; Meira, Ricardo Radaelli; Teixeira, } \\
\text { Adriano Canabarro. Avaliando a Formação de Professores no Contexto do } \\
\text { Pensamento Computacional. Revista Novas Tecnologias na Educação - } \\
\text { RENOTE. v. 16, 2018. Disponível em: } \\
\text { https://seer.ufrgs.br/renote/article/view/89274/51514. Acesso em: } 19 \text { fev. } 2021 .\end{array}$ & [1] \\
\hline $\begin{array}{l}\text { Lira, Leitão e } \\
\text { Castro (2019) }\end{array}$ & $\begin{array}{l}\text { LIRA, Arianny de Sousa; LEITÃO, Darlene Alves; Castro, Juscileide Braga } \\
\text { de. Como o processo de produção de mídias pode contribuir para a formação } \\
\text { docente? Revista Novas Tecnologias na Educação - RENOTE. v. 17, 2019. } \\
\text { Disponível em: https://seer.ufrgs.br/renote/article/view/95850/53869. Acesso } \\
\text { em: } 19 \text { fev. } 2021 \text {. }\end{array}$ & {$[2]$} \\
\hline $\begin{array}{l}\text { Silva, Silva e } \\
\text { França (2017) }\end{array}$ & $\begin{array}{l}\text { Silva, Vladimir; Silva, Luis Lima Da; França, Rozelma. Pensamento } \\
\text { computacional na formação de professores: experiências e desafios } \\
\text { encontrados no ensino da computação em escolas públicas. Anais do Workshop } \\
\text { de Informática na Escola, [S.1.], p. 805-814, out. 2017. Disponível em: } \\
\text { https://br-ie.org/pub/index.php/wie/article/view/72992021. Acesso em: } 19 \text { fev. } \\
2021 \text {. }\end{array}$ & {$[3]$} \\
\hline $\begin{array}{l}\text { Martinelli, } \\
\text { Sakata, Zaina } \\
\text { (2019) }\end{array}$ & $\begin{array}{l}\text { Martinelli, Suéllen Rodolfo; Sakata, Tiemi Christine; Zaina, Luciana } \\
\text { Aparecida Martinez. MultiTACT: uma abordagem para a construção de } \\
\text { atividades de ensino multidisciplinares para estimular o Pensamento } \\
\text { Computacional no Ensino Fundamental I. 2019. Dissertação de Mestrado. } \\
\text { Disponível em: https://repositorio.ufscar.br/handle/ufscar/11199. Acesso em: } \\
27 \text { fev. } 2021 .\end{array}$ & [4] \\
\hline $\begin{array}{l}\text { Oliveira, } \\
\text { Bittencourt e } \\
\text { Trindade (2019) }\end{array}$ & $\begin{array}{l}\text { E. C. Oliveira, R. A. Bittencourt and R. P. Trindade, "Introduction to } \\
\text { Computational Thinking for K-12 Educators through Distance } \\
\text { Learning," 2019 IEEE Frontiers in Education Conference (FIE), Covington, } \\
\text { KY, USA, 2019, pp. 1-9, doi: 10.1109/FIE43999.2019.9028492. }\end{array}$ & {$[5]$} \\
\hline $\begin{array}{l}\text { Reimer et al. } \\
(2018)\end{array}$ & $\begin{array}{l}\text { Y. J. Reimer, M. Coe, L. M. Blank and J. Braun, "Effects of Professional } \\
\text { Development on Programming Knowledge and Self-Efficacy," 2018 IEEE } \\
\text { Frontiers in Education Conference (FIE), San Jose, CA, USA, 2018, pp. 1-8, } \\
\text { doi: 10.1109/FIE.2018.8659041. }\end{array}$ & {$[6]$} \\
\hline
\end{tabular}

Quadro 6. Quantitativo de trabalhos encontrados, filtrados e analisados por base

\begin{tabular}{|c|c|c|c|}
\hline & $\begin{array}{c}\text { String de busca } \\
\text { inicial }\end{array}$ & $\begin{array}{c}\text { Após Critérios de inclusão e } \\
\text { exclusão }\end{array}$ & Após critério de qualidade \\
\hline RENOTE & 5 & 3 & 2 \\
\hline WIE & 1 & 1 & 1 \\
\hline SBIE & 1 & 0 & 0 \\
\hline WCBIE & 3 & 2 & 0 \\
\hline BDTD & 12 & 2 & 0 \\
\hline RBIE & 1 & 0 & 2 \\
\hline IEEE & 62 & 3 & 0 \\
\hline ACM & 22 & 2 & 6 \\
\hline Total & 122 & 13 & \\
\hline
\end{tabular}




\subsection{Qual o contexto, período, coleta de dados e avaliação empregada nas práticas de formação de professores com Pensamento Computacional?}

Observa-se a escassez de trabalhos que tenham foco na formação de professores para atuar com Pensamento Computacional dentro do período pesquisado. Esta questão vai de encontro aos trabalhos de revisão e mapeamento, que consideram o mesmo período, e que apontam a presença de trabalhos com PC para alunos. Desse modo, revelam que os trabalhos para aplicar Pensamento Computacional em sala de aula estão sendo realizados por equipes de pesquisa, promovidos dentro de projetos e eventos e buscam formar os professores em período específico, não havendo prosseguimento das ações de forma periódica. Contudo, emerge seguinte pergunta: Se os professores não estão sendo formados, quem dará continuidade à formação dos alunos? Ela apenas se restringirá àquela ocasião em que uma equipe visitou a escola no intuito de coletar dados para suas pesquisas? Isso é preocupante e descreve uma realidade que necessita de maiores estudos e consequentes análises.

No que concerne aos trabalhos que objetivaram formar os professores, atenta-se para o contexto em que ele foi desenvolvido, nesse caso, híbrido e a distância, o que permite maior flexibilidade para quem participa, posto que a carga horária da maioria dos professores chega a ser exaustiva e não permite que todos façam essas formações. Outro aspecto observado é a ausência da aplicação de atividades desplugadas, o que para um cenário como o brasileiro, onde nem todas as escolas possuem infraestrutura de hardware e software disponível, é prejudicial, dado que outros trabalhos já relatam a necessidade de se aplicar atividades desplugadas por não requerer uso desses recursos.

Não existe um consenso sobre quais teorias devem ser utilizadas para avaliar os professores dentro das formações com PC. Do mesmo modo, observa-se a predominância da elaboração de formulários on-line como instrumento de coleta de dados; não se observa a presença de feedbacks para quem participou dessas formações, após o processo avaliativo. Nesse sentido, infere-se que a formação finaliza antes da resposta ao formulário, pois não existe uma reciprocidade entre participantes e formadores.

Os feedbacks em uma formação de professores são necessários, pois a formação não assume um caráter apenas formativo ou de pesquisa com tempo delimitado, mas que se estende, que prossegue. Assim, permite que os desafios ou dificuldades que foram colhidos naquela formação voltem para os participantes em forma de produto ou proposta de solução.

Quadro 7. Caracterização dos trabalhos selecionados

\begin{tabular}{|c|c|c|c|c|c|c|}
\hline ID & $\begin{array}{c}\text { Modalidade } \\
\text { de ensino }\end{array}$ & Tipo & $\begin{array}{c}\text { Atividades } \\
\text { desplugadas }\end{array}$ & Período & Coleta de dados & $\begin{array}{c}\text { Teorias para a } \\
\text { avaliação }\end{array}$ \\
\hline [1] & Presencial & Curso & Não & $\begin{array}{c}\text { Um } \\
\text { Semestre } \\
\text { letivo }\end{array}$ & $\begin{array}{c}\text { Entrevista } \\
\text { semiestruturada }\end{array}$ & $\begin{array}{c}\text { Análise de conteúdo } \\
\text { por meio de processo } \\
\text { de codificação e nível } \\
\text { de concordância por } \\
\text { meio da ferramenta } \\
\text { dfreelon }\end{array}$ \\
\hline [2] & Híbrido & Oficina & Não & 2 dias & $\begin{array}{c}\text { Observação por } \\
\text { meio de registros } \\
\text { escritos e } \\
\text { fotográficos; } \\
\text { gravações de }\end{array}$ & NÃO DESCREVE \\
\hline
\end{tabular}




\begin{tabular}{|c|c|c|c|c|c|c|}
\hline & & & & & $\begin{array}{l}\text { áudios, análise dos } \\
\text { materiais } \\
\text { produzidos pelos } \\
\text { participantes e } \\
\text { avaliação } \\
\text { diagnóstica on-line }\end{array}$ & \\
\hline [3] & Presencial & Curso & Sim & 4 dias & Questionário & Escala de Likert \\
\hline [4] & Híbrido & Curso & Sim & 5 meses & $\begin{array}{l}\text { Formulários e } \\
\text { avaliações }\end{array}$ & Estatística Descritiva \\
\hline [5] & Distância & Workshop & Não & 5 semanas & $\begin{array}{c}\text { Formulário no } \\
\text { google forms }\end{array}$ & $\begin{array}{c}\text { (Shapiro-Wilk test, } \\
\text { Wilcoxon rank-sum } \\
\text { test , Spearman's rank) }\end{array}$ \\
\hline [6] & Presencial & Curso & Não & 1 semana & Formulário on-line & Alfa de Cronbach \\
\hline
\end{tabular}

\subsection{Quais são os materiais didáticos que estão sendo adotados para formar professores para atuar com Pensamento Computacional?}

Observa-se que os materiais didáticos utilizados se restringem a ferramentas digitais. Cabe salientar, ainda, que até mesmo os trabalhos que propõem desenvolver atividades desplugadas acabam por utilizar recursos pedagógicos plugados em grande parte do tempo. Assim também, não são observados materiais didáticos desenvolvidos exclusivamente para esses tipos de formações. Isso é um fator problemático, visto que para os alunos existem diversos materiais com o objetivo de ensinar Pensamento Computacional em sala de aula, mas, para os professores, não. Nessa lógica, continuando com os questionamentos levantados na RQ1, indaga-se: para quê e para onde os professores estão sendo formados? Que tipo de professores estão querendo formar para atuar com PC? Paralelo à essa questão, surge uma outra inquietação: as formações de professores para atuar com PC estariam apenas servindo como uma forma de aprender a manipular ferramentas? Se sim, o PC não estaria cumprindo o seu papel desde a formação dos professores, pois uma educação para ser libertadora e propiciar o pensamento crítico só se efetua com o oposto dessa estratégia.

Quadro 8. Listagem de materiais didáticos utilizados pelos trabalhos

\begin{tabular}{|c|l|}
\hline ID & \multicolumn{1}{|c|}{ Recursos pedagógicos } \\
\hline$[1]$ & Scratch \\
\hline$[2]$ & Scratch \\
\hline$[3]$ & CS unplugged, LightBot e Scratch \\
\hline$[4]$ & Code.org e Google Classroom \\
\hline$[5]$ & Scratch e Google Classroom \\
\hline$[6]$ & IDLE e Moodle \\
\hline
\end{tabular}

Dentro desse cenário, um argumento bastante utilizado por quem realiza formações de professores com a utilização de ferramentas digitais é que elas propiciam atividades como as narrações de histórias ou jogos que visam trabalhar o empoderamento 
e o desenvolvimento do raciocínio lógico em seus usuários. Esse argumento é bem condizente com as práticas de quem corrobora a primeira fase do Pensamento Computacional (GUARDA; PINTO, 2020), pelo qual só por meio da programação é que se consegue desenvolver habilidades do PC. Contudo, percebe-se que muitas formações se limitam à manipulação de ferramentas.

Nesse contexto, é necessário ir além dessa manipulação, proporcionar espaços de promoção de práticas criativas e críticas por parte dos professores e estratégias para aplicar o Pensamento Computacional em sala de aula, bem como organizar materiais didáticos mais relevantes para estruturar seu fazer pedagógico. Se for uma ferramenta, excelente, mas se não for, que a escolha seja feita por esse professor que participou de uma formação com caráter emancipatório e que consiga compreender o que utilizar ou até mesmo o que vai querer produzir, e que ele saiba produzir.

\subsection{Existe algum material didático para formar professores para atuar com Pensamento Computacional em alguma modalidade de ensino nessas pesquisas?}

Nos trabalhos analisados não foi localizado nenhum estudo que tivesse como enfoque a formação de professores para atuar com Pensamento Computacional na EJA em alguma modalidade específica de ensino. Por consequência, entende-se que não existem, dentre os trabalhos mapeados, nenhum que aborde Pensamento Computacional na formação de professores que atuam em modalidades, sendo uma das lacunas a Educação de Jovens e Adultos, apesar de existirem trabalhos voltados para os alunos (ORTIZ; PEREIRA, 2019).

Essa Research Question vai ao encontro do que argumentam teóricos que estudam a EJA, em específico a formação de seus professores. Isso ocorre por inúmeros motivos, desde tentativas de apagamento da EJA ao longo dos anos na Constituição (Haddad, Di Pierro), a não inclusão na Base Nacional Comum Curricular (BNCC), uma das ações mais recentes. Assim, o que tende a ocorrer na EJA é a situação levantada na RQ3 sobre reutilização de materiais, mas aqui, para formar professores.

\section{Considerações Finais}

Há muito o que se discutir sobre formação de professores em Pensamento Computacional. Isso ocorre por alguns motivos: a) porque a incidência de artigos publicados no âmbito do PC ainda é incipiente na literatura científica; b) porque ainda são raros os trabalhos que apontam a formação de professores para atuar com PC, tanto que foram localizadas poucas pesquisas abordando essas formações; c) porque preocupa-se primeiro em formar alunos; e d) porque quando ocorrem as formações elas estão se resumindo a formar reprodutores de práticas, manipuladores de ferramentas o que não possibilita a autonomia docente e formação do pensamento crítico numa perspectiva freiriana de educação libertadora. Dentre esses quatros pontos, o c) e o d) são bastante problemáticos.

Analisando-se o item c, infere-se que atualmente tem-se pulado uma etapa: a formação docente. O que compromete o andamento da aplicação do PC em sala de aula, visto que se deixa a cargo de pesquisadores a formação desses alunos, enquanto o professor não é considerado nessa relação didático-pedagógica. Assim, se o professor não é formado, o uso de Pensamento Computacional nas salas de aula se encerra após a publicação científica dos resultados dessas pesquisas. 
No contexto do item d, percebe-se que uma formação docente para atuar com PC focada em aplicar apenas ferramentas contradiz a origem da definição do termo PC, cunhada por Wing (2006). Assim também, revela uma seara frágil a incorporação do conceito de construcionismo desenvolvido por Papert (1989) e que sempre acompanha as definições do PC. Portanto, se o professor está aprendendo com práticas da educação bancária, o aprender fazendo não será efetivado, mas sim, reproduzido numa perspectiva meramente instrumental.

Dessa forma, sugere-se que as práticas sigam o pensamento freireano de que não é viável que se aplique em uma formação apenas a manipulação de ferramentas, tampouco que esses professores simplesmente reutilizem materiais didáticos sem a devida adequação para o contexto da EJA, mas que sejam dados subsídios para que esses docentes sejam capazes de refletir, dialogar com seus alunos e construir ferramentas ou materiais didáticos pautados na abordagem do Pensamento Computacional, posto que o papel do professor é a problematização e organização de forma sistematizada do conhecimento que seus alunos entregam de forma desestruturada (Freire 2005).

Apesar das lacunas relatadas, observa-se que existem muitos campos a serem explorados no âmbito da formação de professores, o que é salutar para quem tem interesse em pesquisar sobre Pensamento Computacional na formação docente. Assim também, destaca-se a necessidade de serem desenvolvidos trabalhos com foco na formação de professores da Educação de Jovens e Adultos, pois esta é uma área que tem muito a revelar sobre a realidade da educação brasileira. Por isso, recomenda-se que as formações com professores que atuam na EJA, bem como possíveis materiais didáticos que venham a ser utilizados, sejam direcionados às características de cada modalidade, a fim de se evitar o que já vem acontecendo: adaptações e reproduções.

\section{Referências}

Aman Yadav and Marc Berges. 2019. Computer Science Pedagogical Content Knowledge: Characterizing Teacher Performance. ACM Trans. Comput. Educ. 19, 3, Article 29 (June 2019), 24 pages. DOI:https://doi.org/10.1145/3303770

Barros, Taise T. T.; Reategui, Eliseo Berni; Meira, Ricardo Radaelli; Teixeira, Adriano Canabarro. (2018) Avaliando a Formação de Professores no Contexto do Pensamento Computacional. Revista Novas Tecnologias na Educação- RENOTE. v. 16. Disponível em: https://seer.ufrgs.br/renote/article/view/89274/51514. Acesso em: 19 fev. 2021.

Bell, Tim et al. (2011) Ensinando Ciência da Computação sem o uso do computador. Computer Science Unplugged ORG.

Fernandes, Fabiano; Machado, Veruska. (2017) O estágio na licenciatura em computação como espaço para formação do professor pesquisador de sua prática: um relato de experiência. Anais dos Workshops do Congresso Brasileiro de Informática na

Educação, [S.1.], p. 824, out. Disponível em: https://brie.org/pub/index.php/wcbie/article/view/7468. Acesso em: 19 fev. 2021.

Kaila, E., Laakso, M. and E. Kurvinen, "Teaching future teachers to code Programming and computational thinking for teacher students," 2018 41st International Convention on Information and Communication Technology, 
Electronics and Microelectronics (MIPRO), Opatija, 2018, pp. 0677-0682, doi: 10.23919/MIPRO.2018.8400127.

Kitchenham, B., Charters, S. (2007) Guidelines for Performing Systematic Literature Reviews in Software Engineering, Tech. rep., Technical report, EBSE Technical Report EBSE-2007-01.

Kitchenham, B., Brereton, P. D. Budgen, (2010) The educational value of mapping studies of software engineering literature, 2010 ACM/IEEE 32nd International Conference on Software Engineering, vol. 1, IEEE, 2010, pp. 589-598.

Lira, Arianny de Sousa; Leitão, Darlene Alves; Castro, Juscileide Braga de. Como o processo de produção de mídias pode contribuir para a formação docente? (2019) Revista Novas Tecnologias na Educação- RENOTE. v. 17. Disponível em: https://seer.ufrgs.br/renote/article/view/95850/53869. 19 fev. 2021.

Martinelli, Suéllen Rodolfo; Sakata, Tiemi Christine; Zaina, Luciana Aparecida Martinez. (2019) MultiTACT: uma abordagem para a construção de atividades de ensino multidisciplinares para estimular o Pensamento Computacional no Ensino Fundamental I. Dissertação de Mestrado. Disponível em: https://repositorio.ufscar.br/handle/ufscar/11199. Acesso em: 27 fev. 2021.

Medeiros, S., Martins, C., \& Madeira, C. (2020) Contextualizando as Atividades Desplugadas para Aumentar o Engajamento das Crianças. In: Anais do XXXI Simpósio Brasileiro de Informática na Educação, (pp. 1543-1552). Porto Alegre: SBC. doi:10.5753/cbie.sbie.2020.1543.

Oliveira, E. C.; Bittencourt, R. A. and Trindade, R. P. (2019) "Introduction to Computational Thinking for K-12 Educators through Distance Learning," 2019 IEEE Frontiers in Education Conference (FIE), Covington, KY, USA, 2019, pp. 1-9, doi: 10.1109/FIE43999.2019.9028492.

Pasqual Júnior, Paulo Antônio; Oliveira, Simone de. (2019) Pensamento Computacional: Uma Proposta de Oficina Para a Formação de Professores. Revista Novas Tecnologias na Educação- RENOTE. v. 17. Disponível em: https://seer.ufrgs.br/renote/article/view/95707/53803.

Reimer, Y. J., Coe, Mm., Blank, L. M. and J. Braun (2018) "Effects of Professional Development on Programming Knowledge and Self-Efficacy," 2018 IEEE Frontiers in Education Conference (FIE), San Jose, CA, USA, 2018, pp. 1-8, doi: 10.1109/FIE.2018.8659041.

Sue Sentance, Jane Sinclair, Carl Simmons, and Andrew Csizmadia. 2018. ClassroomBased Research Projects for Computing Teachers: Facilitating Professional Learning. ACM Trans. Comput. Educ. 18, 3, Article 14 (September 2018), 26 pages. DOI:https://doi.org/10.1145/3171129.

Silva, Vladimir; SILVA, Luis Lima Da; FRANÇA, Rozelma. Pensamento computacional na formação de professores: experiências e desafios encontrados no ensino da computação em escolas públicas. Anais do Workshop de Informática na Escola, [S.1.], p. 805-814, out. 2017. Disponível em: https://brie.org/pub/index.php/wie/article/view/7299. Acesso em: 19 fev. 2021.

SOUZA, Isabelle Maria Lima de; RODRIGUES, Rivanilson da Silva; ANDRADE, 
Wilkerson. Explorando Robótica com Pensamento Computacional no Ensino Médio: Um estudo sobre seus efeitos na educação. Brazilian Symposium on Computers in Education (Simpósio Brasileiro de Informática na Educação - SBIE), [S.1.], p. 490, nov. 2016. ISSN 2316-6533. Disponível em: https://brie.org/pub/index.php/sbie/article/view/6730/4617. Acesso em: 27 fev. 2021.

Schulz, J.M.; Schmachtenberg, R.F. (2017) Construindo o Pensamento Computacional: experiência com o desenvolvimento e aplicação de materiais didáticos desplugados. In: Seminário Institucional do PIBID/UNISC, 2017. 